\title{
O autocuidado do doente renal crônico com fístula arteriovenosa
}

\author{
The self-care of chronic kidney patients with arteriovenous fistula
}

El autocuidado de los pacientes renales crónicos con fístula arteriovenosa

Gabriela Aparecida Paz de Castro Barreto ${ }^{1 *}$, Thaís Millena Cardoso de Lima1 ${ }^{1}$, Thainá França Oliveira $^{1}$, Marcela Milrea Araújo Barros ${ }^{1}$, Isabele Cristina Mascarenhas Costa ${ }^{1}$, Gisele da Silva Cardoso Fernandes ${ }^{1}$, Maria de Fátima Cândida da Silva ${ }^{1}$, Maria Michelle Pereira dos Santos ${ }^{1}$.

\section{RESUMO}

Objetivo: Conhecer as práticas do autocuidado entre doentes renais crônicos para a preservação da fístula arteriovenosa. Métodos: Trata-se de uma pesquisa de campo com o escopo descritivo, de abordagem qualitativa a partir do uso da técnica entrevista com roteiro semiestruturado em uma clínica de hemodiálise em um município da região norte do Brasil. Resultados: Na perspectiva do autocuidado, emergiram duas categorias temáticas: sentimentos e dificuldades vivenciados pelos pacientes portadores da fístula arteriovenosa e estratégias de autocuidado para a manutenção da fístula. Os pacientes referem medo da perda da fístula e dificuldades enfrentadas no ambiente social e familiar pelas inquietações com o olhar do outro sobre a sua nova condições de saúde. Apesar de compreenderem a importância do autocuidado, se esquecem da fístula no membro afetado e realizam atividades que exigem uso da força física, limitando suas atividades de vida diária. Conclusão: Evidencia-se que mesmo com conhecimento sobre a temática, os pacientes apresentaram falhas na priorização do autocuidado e o enfermeiro tem papel fundamental no cuidado. Os achados apresentados estimularam as pesquisadoras na elaboração uma ferramenta educativa que se propõe a colaborar com práticas de autocuidado e o aumento da qualidade de vida desses pacientes.

Palavras-chave: Fístulas arteriovenosas, Hemodiálise, Autocuidado, Doença renal crônica.

\begin{abstract}
Objective: To know the practices of self-care among chronic kidney patients for the preservation of arteriovenous fistula. Methods: This is a field research with a descriptive scope, with a qualitative approach, using the interview technique with a semi-structured script in a hemodialysis clinic in a city in the northern region of Brazil. Results: From the perspective of self-care, two thematic categories emerged: feelings and difficulties experienced by patients with arteriovenous fistula and self-care strategies for maintaining the fistula. Patients report fear of loss of fistula and difficulties faced in the social and family environment due to concerns about the other's view of their new health conditions. Despite understanding the importance of self-care, they forget about the fistula in the affected limb and perform activities that require the use of physical strength, limiting their activities of daily living. Conclusion: It is evident that even with knowledge on the subject, patients showed failures in prioritizing self-care and nurses have a fundamental role in care. The findings presented encouraged the researchers to develop an educational tool that aims to collaborate with self-care practices and increase the quality of life of these patients.
\end{abstract}

Keywords: Arteriovenous fistulas, Hemodialysis, Self-care, Chronic kidney disease.

\footnotetext{
${ }^{1}$ Faculdade Interamericana de Porto Velho - UNIRON, Porto Velho - RO.

*E-mail: gabrielanet9090@gmail.com
}

SUBMETIDO EM: 6/2021

ACEITO EM: 6/2021

PUBLICADO EM: 6/2021 


\section{RESUMEN}

Objetivo: Conocer las prácticas de autocuidado en pacientes renales crónicos para la preservación de la fístula arteriovenosa. Métodos: Se trata de una investigación de campo con alcance descriptivo, con abordaje cualitativo, utilizando la técnica de entrevista con guión semiestructurado en una clínica de hemodiálisis en una ciudad de la región norte de Brasil. Resultados: Desde la perspectiva del autocuidado, surgieron dos categorías temáticas: sentimientos y dificultades que experimentan los pacientes con fístula arteriovenosa y estrategias de autocuidado para el mantenimiento de la fístula. Los pacientes informan temor a la pérdida de la fístula y las dificultades que enfrentan en el entorno social y familiar debido a las preocupaciones sobre la visión del otro sobre sus nuevas condiciones de salud. A pesar de comprender la importancia del autocuidado, se olvidan de la fístula en el miembro afectado y realizan actividades que requieren el uso de la fuerza física, limitando sus actividades de la vida diaria. Conclusión: Es evidente que aún con conocimientos sobre el tema, los pacientes mostraron fallas en la priorización del autocuidado y las enfermeras tienen un rol fundamental en el cuidado. Los hallazgos presentados animaron a los investigadores a desarrollar una herramienta educativa que tiene como objetivo colaborar con las prácticas de autocuidado y aumentar la calidad de vida de estos pacientes.

Palabras clave: Fístulas arteriovenosas, Hemodiálisis, Autocuidado, Enfermedad renal crónica.

\section{INTRODUÇÃO}

A Doença Renal Crônica (DRC) é uma problemática na saúde pública mundial e crescente, de alta prevalência e número de óbitos, caracterizada pela degradação progressiva e irreversível de função renal, onde o funcionamento do corpo para manter a homeostasia metabólica e hidroeletrolítica entrará em falência (CLEMENTINO DC, et al., 2018). Essa condição clínica também é considerada emergente, cujo impacto gerado na vida do paciente e de sua família por vezes se sobrepõe aos campos social e econômico, tendo em vista os elevados custos governamentais com o tratamento da doença (GONÇALVES AWGB, et al., 2020).

Dados de 2017 mostraram que, no Brasil, há um total estimado de 126.583 pacientes em tratamento dialítico. Destes, 91,8\% utilizam a hemodiálise como forma de terapia renal substitutiva. Nesse mesmo ano, o número estimado de novos pacientes em diálise era de 40.307 (ROCHA RPF e PINHO DLM, 2019). Para tanto, o diagnóstico precoce da DRC é essencial para que estratégias terapêuticas sejam eficazes, para a prevenção ou retardamento de seu desenvolvimento e o ingresso do paciente na terapia renal substitutiva a diálise ou transplante renal (MARINHO AWG, et al., 2020).

Pacientes em hemodiálise precisam de um acesso venoso calibroso, seguro, permanente e com um bom fluxo sanguíneo, facilitando a saída do sangue para o filtro de hemodiálise, de onde, após ser filtrado, retorna para o paciente (FERNANDES AR, et al., 2018). A Fístula Arteriovenosa (FAV) é um acesso vascular permanente e eficiente, que proporciona duração e segurança no tratamento dialítico. Esse acesso é realizado no centro cirúrgico com anestesia local, onde o cirurgião vascular promove a anastomose, junção da artéria radial e veia basílica ou artéria braquial e veia basílica (MATIAS DMM, et al.,2020).

A FAV mesmo sendo o acesso vascular padrão para a hemodiálise é suscetível à várias complicações, tais como: hipofluxosanguíneo, tromboses, aneurismas, infecções, isquemia e edema de mão ou sobrecarga cardíaca (SILVA NA, et al., 2020). Desde que foi retratada em 1966 a colocação da fístula arteriovenosa autógena (FAV) no punho, ainda é o acesso vascular de primeira escolha para o tratamento de usuários que realizam a hemodiálise, pois, está correlacionado a baixos níveis de complicações e de melhor duração a longo prazo (RODRIGUES AT, et al., 2018).

Para Carvalho L, et al. (2020), é fundamental a realização de educação em saúde pois a doença muda o estilo de vida. Os maiores fatores de risco cardiovasculares são a restrição hídrica, a falta de adesão ao tratamento e o sedentarismo, diminuindo assim, a sobrevida desses pacientes. Esses fatores são variáveis e os profissionais de saúde, que rotineiramente o acompanham, devem conscientizá-los sobre a importância da mudança de hábitos, garantia da durabilidade da FAV e consequentemente, melhor qualidade de vida. 
Pesquisas sobre a participação de pacientes com doenças crônicas no autocuidado mostram que a família, os serviços de saúde e a equipe profissional de enfermagem são os influenciadores dos mecanismos que conduzem ao autocuidado. Fundamentados nesses achados, há a necessidade da criação de relações interpessoais de confiança e o comprometimento entre pacientes, familiares / cuidadores e profissionais de saúde promovendo autonomia e responsabilidade compartilhada em relação ao tratamento proposto (ALMEIDA OAE, et al., 2019).

Nesse sentido, o enfermeiro junto à equipe multiprofissional não deve tratar o paciente como dependente de cuidados, mas sim como motivador do seu autocuidado, entendendo seu tratamento e ajudando a desenvolver o seu próprio plano de cuidados (SANTANA ÉCS, et al., 2019).

Frente às considerações relacionadas ao autocuidado dos doentes renais crônicos, desenvolvemos as seguintes questões que conduzirão esta pesquisa: $O$ que os pacientes conhecem e praticam quanto ao autocuidado com a fístula arteriovenosa? Como a enfermagem pode contribuir com esse processo? Nesta perspectiva, o estudo teve por objetivo de conhecer sobre a prática do autocuidado entre doentes renais crônicos para a preservação da fístula arteriovenosa em uma clínica de hemodiálise em um município da região norte do Brasil.

\section{MÉTODOS}

Trata-se de uma pesquisa de campo com o escopo descritivo de abordagem qualitativa. A metodologia qualitativa tem sido amplamente utilizada nos estudos na área da saúde, pois é capaz de incorporar significados e intencionalidades inerentes aos atos, relações e estruturas sociais dos sujeitos estudados, 0 que possibilita uma análise detalhada das construções e relações humanas. Assim, o pesquisador que utiliza o método qualitativo busca compreender a subjetividade do sujeito a partir de seu contexto (SALVADOR PTCO, et al., 2020).

A amostra do estudo foi composta por pacientes DRC que realizam o procedimento hemodiálise através de FAV. $\mathrm{Na}$ investigação, optou-se pela amostra não probabilística, por conveniência, que é quando o pesquisador seleciona pessoas da população em estudo que se mostrem mais colaborativos ou disponíveis para participar do processo (FREITAG RMK, 2018).

Foram constituídos como critérios de inclusão os pacientes DRC, cadastrados no serviço de nefrologia durante o tratamento de hemodiálise, que fazem uso da FAV como acesso vascular, cujo procedimento estava sendo realizado no período vespertino e que aceitaram participar voluntariamente do estudo mediante a assinatura do Termo de Consentimento Livre e Esclarecido (TCLE). Foram exclusos da pesquisa pacientes que faziam uso de cateter provisório para hemodiálise, participantes com idade inferior a 18 anos e aqueles que realizam tratamento em períodos da manhã ou noite.

O roteiro da entrevista foi composto por 6 (seis) perguntas, cujo momento aconteceu de maneira individualizada durante as sessões de hemodiálise, autorizada pela gestão do cenário de pesquisa. Esta etapa teve duração de aproximadamente 20 (vinte) minutos para cada participante. As entrevistas semiestruturadas foram realizadas no horário vespertino (14h30min às $18 \mathrm{~h} 30 \mathrm{~min}$ ), na clínica de hemodiálise em um município da região norte do Brasil, considerando os horários em que os pacientes se encontravam em um período de três horas e meia a quatro horas para a realização da hemodiálise.

Esse estudo é um recorte de uma pesquisa intervenção oriunda do Trabalho de conclusão de Curso de Graduação em Enfermagem intitulado "O autocuidado dos Doentes Renais Crônicos com Fistula Arteriovenosa: contribuições de Enfermagem". A pesquisa foi submetida ao Comitê de Ética em Pesquisa da União Educacional do Norte Ltda (UNINORTE) em 1 de julho de 2020, sob o parecer no 4.128.804 e CAAE: 31656120.9.0000.8028. O estudo obedeceu aos critérios instituídos pela Resolução 466/12 do Conselho Nacional de Saúde/ Ministério da Saúde, que aprova as diretrizes e normas regulamentadoras de pesquisas envolvendo seres humanos.

Os resultados das entrevistas foram analisados mediante análise de conteúdo. Após a gravação e transcrição das entrevistas na íntegra, elas foram destruídas para manter o sigilo das informações, posteriormente analisadas de acordo com o referencial teórico. Foi analisado através dos recortes das falas 
dos participantes que representam as estruturas de significados, agrupando-se os trechos que expressam motivos em comum referentes à ação. Os nomes dos sujeitos foram apresentados com os seguintes pseudoanônimos: PFA1, PFA2, PFA3, PFA4, PFA5, PFA6, PFA7, PFA8, PFA9, PFA10, PFA11, PFA12, PFA13, PFA14, PFA15.

\section{RESULTADO E DISCUSSÃO}

Fizeram parte do estudo 15 (quinze) pacientes doentes renais crônicos em tratamento de hemodiálise com uso da fístula arteriovenosa. O paciente renal crônico vive diariamente a expectativa de se submeter ao tratamento hemodialítico e, após o término de cada sessão, retornar às suas atividades cotidianas. Acontece que esse tratamento desencadeia sentimentos de limitações que podem refletir nas práticas do autocuidado, gerados pelas restrições decorrentes do tratamento hemodialítico e da manutenção da FAV.

Isso nos permite discutir elementos contextuais relacionados à fístula arteriovenosa e, a partir das narrativas, buscar evidências científicas que reflitam na implementação de uma assistência de enfermagem qualificada. A partir da análise de modo empírico, tendo como bases semelhantes e convergentes, emergiram duas categorias temáticas que colaboraram para organizar o processo de apresentação dos resultados: Sentimentos e dificuldades vivenciados pelos pacientes portadores de FAV e, Estratégias de autocuidado para a manutenção da Fístula Arteriovenosa.

\section{Sentimentos e dificuldades vivenciados pelos pacientes portadores da FAV}

Conforme relatos quando questionados sobre os sentimentos vivenciados por ser portador de FAV, observamos que os doentes renais portadores da Fistula Arteriovenosa apresentaram dificuldades enfrentadas no ambiente social e familiar, relacionadas às inquietações com o olhar do outro sobre a sua nova condições de saúde / doença. Ao experimentarem essa situação de adversidade, as marcas em seu corpo os caracterizam como um ser diferente no convívio social.

"No início eu ficava meio assim porque todo mundo ficava olhando, 'o que é isso?"” (PFA 1).

"É bem complicado hoje em dia porque é muito visível, então onde você chega as pessoas, nem olham para o seu rosto, e sim direto pra fístula" (PFA 4).

Silva NA, et al. (2020) destacam que ao se deparar com essa necessidade o paciente passa por um processo de rejeição e de aceitação, podendo interferir no seu estado emocional e psicológico, gerando algumas consequências na forma de enfrentar a doença e o tratamento.

Em estudo realizado por Silva DM, et al. (2018) os participantes portadores da FAV revelaram que esse acesso venoso deixa marcas no corpo que alteram a estética corporal, tornando o corpo 'imperfeito'. Essas alterações geram baixa autoestima, atraem o olhar do outro e causam constrangimentos naquele que tem 0 corpo marcado. Esse, por sua vez, reage escondendo a fístula, sem a qual não há vida.

Quando questionados sobre o autocuidado os doentes renais crônicos expuseram a importância de seguir corretamente os cuidados com a fístula e relataram o receio pela possibilidade de perda do acesso e o retorno para o cateter venoso, como um retrocesso no tratamento:

"É de grande importância porque é aqui que vou fazer a minha hemodiálise, porque senão, eu vou ter que usar cateter e eu tive a experiência e não foi boa" (PFA 1).

"Muito importante porque é muito ruim ficar com cateter, no começo eu não queria fazer a fístula devido a estética” (PFA 4).

"Se você perder, vai voltar pro cateter e é ruim demais" (PFA 7).

A FAV é considerada como acesso padrão-ouro para hemodiálise é um acesso permanente com um quantitativo menor de complicações quando comparado aos cateteres venosos centrais, que aumentam em $50 \%$ a taxa de mortalidade e que podem deteriorar o sistema venoso dos pacientes (estenoses centrais, tromboses), podendo dificultar a sua confecção posteriormente (CORREIA BR, et al., 2021). 
Considerando que os pacientes com doença renal crônica são dependentes dos acessos para a realização da terapia dialítica, o prolongamento do tratamento diminui as chances de sua funcionalidade. Essa situação reflete em sentimentos, como o medo relacionado à perda do acesso vascular e consequentemente a interrupção do tratamento (SILVA PFC, et al., 2017).

Embora alguns pacientes relatem que não sentem vergonha do corpo, ainda enfrentam situações de constrangimento não apenas de desconhecidos, mas também de seus familiares. Esses sentimentos surgem devido às mudanças na identidade pessoal gerando conflitos emocionais e auto aceitação:

"Já vieram me perguntar se tenho câncer, por que eles não sabem do assunto, mas eu evito na minha família" (PFA 4).

"Eu gosto de usar mais blusa de manga longa, porque as pessoas perguntam o que é e olham diferente pela estética do braço, eu fico chateada com isso" (PFA 2).

"No começo é ruim, as pessoas perguntam, por causa do 'calombo', mas isso a gente vai levando, em casa minhas filhas no começo ficavam abismada comigo "pai isso ai é normal?" Agora não, já costumei, é só ter cuidado" (PFA 6).

Rocha MAM, et al. (2019) descrevem que as dificuldades que podem acontecer ao paciente com IRC vão interferir em seu ambiente social e cultural, em algumas situações em suas crenças, por isso o apoio de familiares, profissionais envolvidos com a recuperação e cuidado desse paciente é essencial. É importante que o paciente receba apoio social tanto da família quanto da equipe multiprofissional, o que auxilia na recuperação ou nos momentos de recaída.

Segundo Neto JMR, et al. (2016) as emoções e sentimentos dos pacientes que surgem com a implantação da fístula arteriovenosa, são bastante diversificados. Em um estudo realizado, no setor de Nefrologia de um Hospital da rede privada do município de João Pessoa-PB. Com doentes renais crônicos seus depoimentos revelaram sentimentos de irritação pela doença e fístula, tristeza, sofrimento, atualmente superados em sua maioria.

Carneiro CT, et al. (2018) enfatizam que o sentimento de vergonha associado à aparência física, demonstrado nas falas dos pacientes da clínica em hemodiálise, evidenciam que os pacientes renais têm medo de imaginar o que as pessoas diriam ou perguntariam sobre o uso do cateter e alterações no braço onde se localiza a fístula, o que contribui para autoimagem negativa e o surgimento de sentimentos de inferioridade.

Outros relatos registrados referem-se às dificuldades vivenciadas e sua relação às limitações nas atividades diárias de vida.

"Sou dona de casa e tem muitas coisas que eu não faço ou evito, para limpar a casa eu tive que comprar um rodo adequado para não torcer o pano, roupa eu não lavo, tenho a máquina e só estendo" (PFA 1).

"A dificuldade é muito grande porque eu não posso fazer muitas coisas que quero, fico impossibilitado de não mexer com ela, tem que tá trabalhando só com a mão esquerda" (PFA 6).

Fernandes D, et al. (2020) enfatizam que a HD acarreta diversos tipos de limitações nos indivíduos submetidos a esse tratamento de alta complexidade. As limitações físicas e mentais com a evolução do tempo de tratamento podem diminuir as complicações hidroeletrolíticas, a percepção do medo, contribuindo para o enfrentamento da doença, as restrições por ela impostas, bem como melhorar a percepção do estado geral de saúde.

Nessa perspectiva, Ribeiro WAR, et al. (2020) ressaltam que essas limitações nas atividades diárias, especialmente de ordem física, aumentam com o avanço da idade, pois os idosos apresentam a fragilidade decorrente do processo de envelhecimento e estão mais propícios à ocorrência de múltiplas comorbidades. Na maioria dos casos, essa terapia cria frustração e limitações porque vem com algumas proibições. 


\section{Estratégias de autocuidado para a manutenção da fístula arteriovenosa}

Na perspectiva do autocuidado com a fístula, observamos que apesar de compreender a sua importância, em situações cotidianas, se esquecem da FAV no membro afetado e realizam atividades que exigem uso da força física:

"Às vezes eu esqueço e pego peso" (PFA 9).

"A gente acaba esquecendo pegando um pesinho uma forcinha, garrafão de água" (PFA 15).

De acordo com Santana ÉC (2019) os cuidados como não pegar peso, evitar pancadas e nem fazer esforço com o braço da FAV visam impedir que o braço da fístula sofra qualquer tipo de trauma, o que pode interromper o fluxo sanguíneo, levando a uma trombose no acesso, ocasionando grandes transtornos e prejuízos para o paciente.

Quando questionados sobre o autocuidado, percebeu-se durante as respostas dos participantes que os DRC apresentaram dificuldade em seguir as estratégias de autocuidados em seu domicílio, sendo perceptível que os mesmos compreendem que ao dormir em cima do braço podem gerar complicações.

\section{"[...] também durmo por cima do braço" (PFA 9). \\ "[...] e ás vezes e inevitável a gente deita em cima do braço, tem que ter muito cuidado" (PFA 4).}

Magalhães VPR, et al. (2020) apontam que parte do autocuidado pelo paciente, deve-se proteger a fístula arteriovenosa contra traumatismos e evitar, no próprio membro, a realização de esforços físicos e dormir sobre o braço utilizado por exemplo. Desse modo, não se interrompe o fluxo sanguíneo na região, o que diminui a ocorrência de complicações como dilatações, ruptura espontânea, hematomas e trombose da fistula arteriovenosa.

Os DRC expuseram a falta de interesse em praticar os cuidados após as sessões de hemodiálise em seu domicilio. Dentre os cuidados necessários para manter a FAV em bom funcionamento cita-se o cuidado com o curativo. É importante enfatizar que as orientações prestadas não dependem apenas das ações aplicada pela equipe de saúde, mas, pela atenção do paciente após a hemodiálise.

\section{"Um curativo tirado antes da hora, a gente chega em casa e faz outro [...], às vezes a gente não cuida" (PFA 15).}

Os curativos devem ser retirados 6 (seis) horas após o término da hemodiálise, exigindo uma reorientação dos pacientes quanto a esse cuidado, visto que a grande maioria permanece com o curativo por 24 horas, 0 que é inadequado, uma vez que não há dados na literatura que apontem para essa prática (CLEMENTINO DC, et al., 2018).

Mudança de hábitos ordenam, de modo consequente, diminuição das taxas de infecção, menos custos, dessa forma evitará inserção de novos cateteres ou confecção de novas (FAV). Levar em consideração que o usuário é o agente fundamental do próprio tratamento, o enfermeiro intervém além da assistência, sendo assim o educador com o papel primordial na orientação da hemodiálise e dos cuidados com acessos e, dessa maneira facilitando a promoção do autocuidado (FREITAS LR, et al., 2019).

Silva S, et al. (2020) discorrem que o cuidado de enfermagem visa promover maior durabilidade da fístula e compreende ações como manter o braço elevado, evitar curativos circunferenciais apertados, realizar avaliação diária do fluxo sanguíneo e realizar exercícios de compressão manual. Além disso, durante todo o período de uso da fístula, alguns cuidados devem ser tomados, como compressão adequada para hemostasia após a diálise, poupando o uso do membro de acesso evitando grandes esforços, infusões venosas e aferições da pressão arterial.

A partir do estudo realizado foram descobertos os elementos para a construção de possíveis soluções, de forma criativa e crítica. O que precisa e pode ser feito para que o problema seja resolvido? As hipóteses são construídas após o estudo, como conclusão da compreensão da realidade, analisar o problema de todos os ângulos possíveis (VIEIRA MNM e PINTO MPP, 2015). 


\section{CONCLUSÃO}

Constatou-se neste estudo que os doentes renais crônicos portadores da fistula arteriovenosa, mesmo com conhecimento sobre a via de acesso, apresentaram falhas na priorização do autocuidado. A partir das análises dessa pesquisa como propositura e contribuição da academia de enfermagem nos processos de educação em saúde, as pesquisadoras foram estimuladas a elaborar uma ferramenta educativa do tipo cartilha para ser implementada e validada em estudo posterior. Pois a educação em saúde é considerada um processo dinâmico e colaborativo, contribuindo para a compreensão da importância do autocuidado, a escuta ativa e a capacidade de estimular hábitos de maneira adequada respeitando-se a individualidade. Lacunas no conhecimento encontrados abrem espaço para impulsionar novos estudos acerca da temática voltadas para a participação da família e contribuições da enfermagem ao paciente com a fístula arteriovenosa.

\section{REFERÊNCIAS}

1. ALMEIDA OA, et al. Envolvimento da pessoa com doença renal crônica em seus cuidados: revisão integrativa. Ciência \& Saúde Coletiva [online], 2019; 1689-1698.

2. CARNEIRO CT. Vivências de adolescentes e jovens diagnosticados com doença renal crônica. Rev. Aten. Saúde, 2018; 2429.

3. CARVALHO Jl, et al. Fatores de Risco para Pacientes com Falência Recorrente de Fístula Arteriovenosa. Revista de Pesquisa: Cuidado é Fundamental Online, 2019; 1188-1193.

4. CLEMENTINO DC, et al. Pacientes em hemodiálise: importância do autocuidado com a fístula arteriovenosa. Revista enfermagem UFPE online, 2018; 1841-1852.

5. CORREIA BR, et al. Avaliação clínica da maturação da fístula arteriovenosa para hemodiálise: revisão de escopo. Acta Paul Enferm, 2021; 34.

6. CORREIA BR, et al. Utilização do exame físico na avaliação da funcionalidade das fístulas arteriovenosas para hemodiálise. R. pesq.: cuid. fundam. Online, 2021; 177-184.

7. FERNANDES AR, et al. Identificação da fístula arteriovenosa e suas complicações pelos enfermeiros dos serviços de entrada de Cáceres - MT. Rev. APS, 2018; 408-417.

8. FERNANDES D, et al. Qualidade de vida de pacientes em terapia renal substitutiva: uma análise da doença renal crônica e perfil populacional de risco. Revista Eletrônica Acervo Saúde, 2020; 12(12): 4759.

9. FREITAS, LR, de et al. Cartilha para o paciente em diálise renal: cuidados com cateteres venosos centrais e fístula arteriovenosa. Rev. Bras. Enferm. [online], 2019; 896-902.

10. FREITAG RMK. Amostras sociolinguísticas: probabilísticas ou por conveniência?. Revista de estudos da Linguagem, 2018; 667-686.

11. GONÇALVES LM, et al. Cuidados de enfermagem a clientes com fistula arteriovenosa: uma revisão integrativa da literatura. Revista de Pesquisa: Cuidado é Fundamental Online, 2020; 444-449.

12. MAGALHÃES VAR, et al. Fístula Arteriovenosa na insuficiência renal crônica: cuidados e complicações. Braz. J. Hea. Ver., 2020; 2000-2007.

13. MARINHO AWGB, et al. Prevalência de doença renal crônica autorreferida em adultos na Região Metropolitana de Manaus: estudo transversal de base populacional, 2015. Epidemiologia e Serviços de Saúde [online], 2020.

14. MATIAS DMM, et al. Cuidado individual domiciliar de pacientes com fístula arteriovenosa. Rev. enf. UFPE on line, $2020 ; 1-7$.

15. MOURA AS, et al. Jogo dramático e a saúde mental do docente. Com. Ciências Saúde, 2018; 29: 265-272.

16. NETO JMR, et al. Fístula arteriovenosa na perspectiva de pacientes renais crônicos. João Pessoa-PB. Enferm. Foco, 2016.

17. SALVADOR PTCO, et al. Estratégias de coleta de dados online nas pesquisas qualitativas da área da saúde: scoping. Revista Gaúcha de Enfermagem [online], 2020; 41.

18. SANTANA EC, et al. Perfil dos pacientes submetidos a tratamento hemodialítico em uma clínica em Teresina. Rev Fun Care Online, 2019; 11(1): 142-146.

19. RIBEIRO WAR, et al. Repercussões da hemodiálise no paciente com doença renal crônica: uma revisão da literatura. Revista Pró-UniverSUS, 2020; 11(1): 88-97

20. ROCHA MAM, et al. O bem-estar de pacientes renais crônicos durante o tratamento com hemodiálise e diálise peritoneal. Revista Eletrônica Acervo Saúde, 2019; Sup.21: 670.

21. ROCHA RPF, PINHO DLM. Ocorrência de eventos adversos em unidades públicas de hemodiálise. Enfermería Global, 2019; 18(3): 1-34.

22. RODRIGUES AT, et al. Avaliação de variáveis associadas à permeabilidade de fístulas arteriovenosas criadas por um nefrologista para fins de hemodiálise. J. Bras. Nefrol., 2018; (4): 326-332.

23. SILVA AN, et al. O uso de metodologia ativa no campo das Ciências Sociais em Saúde: relato de experiência de produção audiovisual por estudantes. Interface (Botucatu), 2020.

24. SILVA DM, et al. O corpo marcado pela fístula arteriovenosa: um olhar fenomenológico. Rev. Bras. Enferm., Brasília, 2018; (6): 2869-2875.

25. SILVA PFC, et al. Influência dos acessos vasculares na autoimagem e sexualidade dos pacientes em hemodiálise: contribuição para enfermagem. Cienc Cuid Saude, 2017; 16(1).

26. SILVA RS, et al. Assistência de enfermagem na manutenção do acesso vascular arteriovenoso de pacientes renais crônicos em hemodiálise: uma revisão narrativa. Revista Eletrônica Acervo Saúde, 2020; Sup.44: 2956.

27. SANTANA EC, et al. Perfil dos pacientes submetidos a tratamento hemodialítico em uma clínica em Teresina. Rev Fun Care Online, 2019; 11(1):142-146.

28. VIEIRA MNM, PINTO MPP. A Metodologia da Problematização (MP) como estratégia de integração ensino-serviço em cursos de graduação na área da saúde. Medicina (Ribeirão Preto), 2015; (3): 241-248. 